\title{
Rat feeding studies with genetically modified maize - a comparative evaluation of applied methods and risk assessment standards
}

\author{
Hartmut Meyer ${ }^{{ }^{*}}$ and Angelika Hilbeck ${ }^{1,2}$
}

\begin{abstract}
A 2-year rat feeding study with genetically modified NK603 maize sparked an international scientific and public debate as well as policy responses by the European Commission. The European Food Safety Authority (EFSA) evaluated the study as defective based on conceptual and methodological shortcomings by retroactive application of the recommendations of its recent guidance on 90-day feeding studies. Our comparative analysis of the three relevant NK603 publications, including a 90-day feeding study of Monsanto, showed that all of them satisfy or fail to satisfy the EFSA evaluation criteria to a comparable extent; the rejection of only one of the papers is, thus, not scientifically justified. We also show that EFSA's criteria are not standard practice in 21 other rat feeding studies lasting at a minimum of 12 months. The review reveals critical double standards in the evaluation of feeding studies submitted as proof of safety for regulatory approval to EFSA. We specifically argue that the current approach to declare statistically significant differences between genetically modified organisms and its parents as 'biologically irrelevant' based on additional reference controls lacks scientific rigor and legal justification in the European Union (EU) system. Only recently, the EU authorities started building up an implementing system based on its own legislation and supportive of the EU approach to risk assessment in the context of technology assessment. Until these issues are resolved, we do not expect that neither the public nor the scientific debate will subside.
\end{abstract}

Keywords: EFSA; Genetically modified food; Long-term studies; Monsanto; NK603 maize

\section{Review} Introduction

In 2007, Séralini et al. [1] re-analyzed the data of a 90day rat feeding trial conducted by Monsanto Company and submitted to the European Food Safety Authority (EFSA) to demonstrate the safety of their genetically modified (GM) MON863 maize for food and feed import in the European Union (EU). In their re-analysis, the authors stated that the data generated by Monsanto Company revealed signs of hepatorenal toxicity. The authors concluded - in disagreement with Monsanto Company and EFSA - that 'Longer experiments are essential in order to indicate the real nature and extent of the possible pathology.' and that 'With the present data, it cannot be concluded that GM corn MON863 is a safe

\footnotetext{
* Correspondence: hmeyer@ensser.org

${ }^{1}$ European Network of Scientists for Social and Environmental Responsibility (ENSSER), In den Steinäckern 13, 38116 Braunschweig, Germany Full list of author information is available at the end of the article
}

product.'. This work sparked a controversial debate in the public and in the political, regulatory, and scientific communities regarding the disputed safety of that particular GM crop and GM food and feed testing in general. The controversy eventually elicited a policy response of the European Commission's Directorate-General for Health and Consumer Safety. In February 2013, an implementing regulation on applications for authorization of GM food and feed was adopted, which prescribes that for future GM crop applications, 90-day feeding trials are mandatory [2].

Meanwhile, Séralini et al. [3] published a report about a 2-year toxicology study with rats testing Monsanto's NK603 glyphosate-tolerant GM maize and its Roundup co-technology. The authors stated that female rats in the treated group died earlier and more often than in the control group. Treated female rats also developed mammary tumours earlier and more often than control female rats. Treated male rats showed higher levels of liver congestion and necrosis as well as kidney nephropathies. The 
authors underlined that $76 \%$ of altered biochemistry parameters were kidney related. The Séralini study elicited an immediate public debate showing a wide range of opinions (which also precipitated in scientific journals, e.g. [4-6]). As a reaction to this debate, the European Commission decided to provide funding for an own 2-year study on NK603 maize [7].

In this article, we evaluate the first response [8] by the EU risk assessment body, EFSA, and the voiced criticism that focuses on the underlying methodological issues. In our analysis, we will leave out the critical issue of applied statistical evaluations of the feeding trial, as this requires other expertise.

EFSA [8] assessed the study [3] with regard to five criteria: study objectives, study design, feed and treatment formulation, statistical methods, and endpoint reporting. These criteria are derived from the recently published 'EFSA guidance on conducting repeated-dose 90-day oral toxicity study in rodents on whole food/feed' [9]. EFSA underlined in its accompanying press release that their analysis focused on the methodology rather than on the outcomes of the Séralini publication. EFSA concludes that the study results are inadequately presented with omission of key details of the design, conduct, analysis, and reporting. Due to its insufficient scientific quality the study would be of no relevance for risk assessments of neither glyphosate nor NK603 maize.

Due to the increasing scientific and public interest, EFSA decided to make available all documents related to the NK603 risk assessment and approval on the internet $[10,11]$. Before, access to these documents was only possible upon individual request for information to EFSA. This still holds true for all other approval applications of other GM organisms (GMOs). Included in the NK603 dossier is the Monsanto Technical Report MSL-17555 [12] describing their 90-day rat feeding study and including the raw data. For its second and final response, EFSA [13] primarily added reactions of EU Member States but did not expand or change the substantive issues published previously.

In order to alleviate this result-triggered, selective application of evaluation criteria and offer an objective evaluation of the applied methodologies in other feeding trials regardless of their outcomes, in particular those on which EFSA based its safety conclusions, we carried out a comparative analysis with the following objectives:

1. To evaluate how Monsanto's technical study [12] and the subsequent peer-reviewed publication of that study's data by Hammond et al. [14], as part of the application for EU wide regulatory approval of NK603 maize met the criteria EFSA applied so far only to the Séralini et al. study [3]. We also include the reply by Séralini et al. [15] on the voiced criticism, which gives additional information on study details.

2. To evaluate the degree of rigor and scrutiny EFSA applied in its assessment of the Monsanto study [12] and Hammond et al. [14] as elements of the NK603 risk assessment procedure. The two scientific opinions of the EFSA Panel on Genetically Modified Organisms on NK603 $[16,17]$ serve as the basis for our analysis.

3. To evaluate how 21 other long-term, peer-reviewed rat feeding studies for comparable purposes but testing other substances would fulfil EFSA's criteria. These additional studies were selected for their methodological criteria being similar to the Séralini study, e.g. a duration of 12 months or longer, using SD rats and performing toxicology sometimes in combination with carcinogenicity tests.

\section{Comparative analysis of the application of the EFSA criteria} The three NK603 publications discussed by us were written - and hence, experiments were planned - before EFSA published its 2011 Guidance on 90-day feeding trials. While the retrospective application of new standards (i.e. study methods) as undertaken by EFSA [8] is debatable, it is broadly accepted that risk assessment informing regulatory decision-making should be based on the latest state of knowledge and standards. A re-evaluation of the applied methods of relevant, older studies is justified if applied to all such relevant studies - but meaningless or even counterproductive when applied selectively to one study or a subset of studies only triggered by their reported (here adverse) effects. We consider such a selective re-evaluation as inappropriate. We note that the main responsibility for EFSA's recent work lies with the European Commission that in its mandate to EFSA singles out the Séralini study for review [17].

\section{EFSA criterion: study objectives}

EFSA [8] notes with regard to Séralini et al. [3] that the 'study objectives are unclear'. EFSA further clarifies that 'If a specific guideline is chosen and followed, then the objectives are inherently defined in the guideline.' To clarify what 'inherently defined' objectives entail, we analyzed the four Guidelines of the Organization for Economic Co-operation and Development [18-21] discussed in this context (OECD nos. 408, 451, 452, 453, respectively). While the three latter Guidelines revised in 2009 give clear objectives, Guideline 408 on 90 -day oral toxicity originally adopted in 1981 and revised in 1998 lacks such clarity. Guideline 408 is the guideline of choice by Monsanto Company for their 90-day rat feeding trial.

Open issue according to EFSA: 'The study objectives need to be clearly stated a priori in the study protocol.' 
Séralini et al. [3] stated that 'Guideline 408 of the Organization for Economic Co-operation and Development (OECD) was followed by some manufacturers for GMOs even if it was not designed for that purpose. We have explored more parameters and more frequently than recommended in this standard in a long-term experiment.' The authors explained that they added biochemical and haematological measurements using 10 rats per group as described in the OECD Guideline 453. This apparent combination of elements of two OECD guidelines and the addition of more parameters did not qualify as proper description of the objectives of the work by EFSA.

In its technical 90-day study, Monsanto tested a lowdose group (LD 11\% NK603) and a high-dose group (HD 33\% NK603) versus two parental control groups and six non-parental, so-called reference controls for 13 weeks. Blood and urine biochemical parameters, amongst others, were measured at an interim time point after 4 weeks and at termination of the study. The statements by Monsanto [12] and later by Hammond et al. [14] to have adapted the OECD Guideline 408 were considered by EFSA to be sufficient. Neither the nature nor the consequences of the adaptations made to the requirements of OECD Guideline 408 were explained in these two publications or in the scientific opinions of EFSA Panel on Genetically Modified Organisms [16,17].

Since OECD Guideline 408 [18] in contrast to the more recent OECD guidelines does not provide clear objectives, it is doubtful whether a mere reference to this guideline as provided by Monsanto suffices EFSA's new standards on study objectives. We conclude that regarding this criterion, the Séralini publication provides at least the same quality in terms of clarity and explanation as the Monsanto and Hammond studies.

\section{EFSA criterion: study design}

The study design applied by Séralini et al. [3] triggered two main criticisms by many commentators including EFSA [8]: (a) the number of rats per group and (b) the choice of the strain of rats.

A. EFSA [8] states: '[g]iven that Séralini et al. (2012) conducted a two-year study, it is unclear why an OECD guideline suitable for a 2-year chronic toxicity or carcinogenicity study (i.e. OECD 451, OECD 452 , or OECD 453) was not adhered to.' Séralini et al. [3] used 10 rats per group as recommended by OECD Guideline 453 [19] for the chronic toxicity phase. But OECD Guideline 453 also clearly states that this lower number is only justified when a carcinogenicity phase with 50 rats per group is run in parallel to support the interpretation of the toxicity data. Later, Séralini et al. [15] argued that the OECD
Guideline 452 in its 1981 version [22], which was applicable for the 2-year toxicology study conducted from 2008 to 2010, advised to only use 10 rats out of the 20 per group for blood and urine sampling and analysis. The recommendation to use at least 10 rats per group for biochemistry analysis at the chosen time points is maintained in the current version of Guideline 452.

Monsanto [12] used 20 rats per sex and group, while the actual values for blood chemistry, haematology and qualitative, and quantitative urine analyses were based on samples from 10 rats per sex and group, thus, comparable to the approach of Séralini et al. [3]. Nowhere in the two versions of Guideline 452 or in the Monsanto study, a method for how to select the 10 animals out of the 20 animals per group for the biochemistry tests is specified.

B. Regarding the choice of SD rats, EFSA notes that this "strain of rats chosen is known to be prone to development of tumours over their life [...] This is neither taken into account nor discussed in the Séralini et al. (2012) publication." A discussion on the choice of rats with regard to their tendency to develop tumours is not relevant for 90-day studies. Nevertheless, EFSA recommends stating reasons for the choice of the strain in its new guidance on 90day feeding trials.

\section{Open issue according to EFSA: 'The biological} relevance of the rat strain used should be justified with respect to the design choices.'

Neither Séralini et al. [3] justify the choice of SD rats nor do Hammond et al. [14]. Monsanto [12] explains that " $\mathrm{t}$ ]he rat was selected for the study since this species has been traditionally used to assess the safety and wholesomeness of food.' This pragmatic explanation underlines the customary use of SD rats in such trials which of course extends also to the Séralini study but does not give a scientifically sound justification for its use with regard to the design choice as implied by EFSA's wording [8].

In (Additional file 1: Table S1), we present information on the actual use of SD rats in peer-reviewed, long-term studies and the types of information researchers usually provide and scientific journals require on the choice of animals. According to the information available, SD rats were chosen as standard test organisms by the two largest toxicity/carcinogenicity research projects worldwide and at least 21 long-term studies:

\section{The National Toxicology Program of the US} Department of Health and Human Services uses this strain in its 2-year studies, after in-depth discussions 
on the suitability and advantages of the SD rat over previously used strains [23].

2. The European Ramazzini Foundation for Oncology and Environmental Sciences (Italy) uses SD rats in its Ramazzini Foundation Cancer Program since more than 40 years [24].

Contrary to many media reports based to a large extent on scientists' personal opinions posted by the Science Media Centre [25], SD rats are used routinely in long-term toxicology and carcinogenicity studies. The rational to do so is that (additional) carcinogenic effects of test substances can be detected more effectively in rats that develop tumours at a relevant rate within the time span of a 2-year trial. Actually, the prime issue of an informed debate would not be so much the choice of the strain of rats but the choice of the numbers of rats depending on the strain used. The OECD Guideline 116 [26] on the conduct of long-term carcinogenicity studies states that '[f] or strains with poor survival such as Sprague-Dawley rats, higher numbers of animals per group may be needed in order to maximize the duration of treatment (typically at least 65/sex/group).' The US Food and Drug Administration (US FDA) [27] recommends for carcinogenicity tests with strains with known problems in survivorship that the researchers need to ensure that at least 25 rats from those used initially remain alive at the end of the 2-year trial. For long-term toxicity trials as conducted by Séralini et al. [3], no specific recommendations are given by the US FDA. With regard to the recommended number of 10 rats per toxicological test in OECD Guideline 452 [20], the above recommendation of FDA should be applied to ensure that in average, more than 10 rats would be available for terminal testing. The reported survival rates of the control rats in Séralini et al. [3] (70\% for males, 80\% for females) lie at or above the upper range presented in available literature on survival rates of SD rats in 2-year studies [28-31].

We conclude that with regard to the choice of rats, a pragmatic approach was followed by Monsanto, Hammond et al. [14] and also Séralini et al. [3]. Since the Séralini group had set out to repeat the 90-day feeding trial by Monsanto but to differ with regard to the extended testing time and parameters measured, the choice of rat was appropriate in order to allow for comparability with regard to this factor. We also conclude that the SD rat strain used is a standard organism for toxicity and carcinogenicity tests. A rejection of the validity of the Seralini study based on the choice of rats would therefore either be based on a lack of familiarity with the scientific field. Or these criteria would have to apply to all carcinogenicity trials conducted to date with this rat strain and, thus, leave us with hardly any valid carcinogenicity study. Criticism of the Seralini study based on the lack of a scientific explanation of the choice of the rat strain would similarly apply to the Monsanto and Hammond publications.

\section{Open issue according to EFSA: 'Suitable controls for all treatment groups are not present.'}

None of the discussed OECD guidelines was developed for the testing of whole foods but rather for testing the toxicity of isolated chemicals when feeding rats with standardized, nutritionally balanced feed. In most cases, the test substance is administered separately from the normal feed or mixed with the feed in a minor volume. As long as the addition of the test substance to the feed does not alter significantly the relative concentration of the feed components, a single control with zero test substance is sufficient. If the test substance is an inherent component of the feed as in the NK603 feeding trial and the different test concentrations are produced through varying percentages of GM and non-GM maize varieties in the feed, appropriate controls are necessary according to EFSA [9]. Hammond et al. [14] and Monsanto [12] report that all test groups received feed containing 33\% maize with a different percentage of GM versus nonGM maize. Séralini et al. [3] did not report whether they had adjusted the total maize content to $33 \%$ in those batches containing $11 \%$ or $22 \%$ GM maize. To clarify, Séralini et al. [15] informed that all feed batches were mixed to contain $33 \%$ maize in total.

We conclude that based on the information given by Séralini et al. [15], this open issue is closed.

\section{Open issue according to EFSA: 'Measures taken to} reduce the risk of bias (e.g. blinding) are not reported.'

While EFSA [8] has noted that Séralini et al. [3] did not report on measures taken to reduce bias in their experiments, we note that also neither Hammond et al. [14] nor Monsanto [12] reported such measures. Blinding measures are not requested by any of the abovementioned OECD guidelines. However, Séralini et al. [15] explained that anatomopathology and biochemical measures were indeed performed in a blinded manner.

The issue of blinded evaluation was promoted by EFSA when it presented its draft [32] of the abovementioned Guidance on a 90-day oral toxicity study for public consultation. EFSA's approach was rejected by many commentators from pathology sciences and industry [33] with reference to the 'Best Practices Guideline: Toxicologic Histopathology' of the Society of Toxicologic Pathology [33]. This guideline explains that unblinded evaluation is regarded as the appropriate approach in the first round of pathological observations because it 
'allows the pathologist to intensely focus the histopathologic evaluation and to find important, and sometimes subtle, differences between the tissues of treated and untreated animals'. The guideline recommends that blinded evaluations should be applied in the re-evaluation of findings in specific tissues and when samples with a defined spectrum of lesions due to a known toxic syndrome are investigated. These recommendations to not apply blinding as a general measure were reflected in EFSA's report on their public consultations [34] and in the final EFSA Guidance on 90-day oral toxicity study. It is unclear why EFSA [8] requested blinding measures in the context of the Séralini study but did take no issue of this in its positive opinions on the NK603 dossiers including Monsanto's rat feeding studies.

We conclude that a double standard was applied with regard to the criterion of blinding. We also conclude that EFSA's approach does not reflect the previously published opinions of various experts in the field.

\section{EFSA criterion: feed and treatment formulation}

Open issue according to EFSA: 'The appropriateness and comparability of the diets cannot be assessed as critical information about their composition is not reported.'

Séralini et al. [3] did not present their data on the composition of the feed but informed that 'all feed formulations consisted in balanced diets, chemically measured as substantially equivalent except for the transgene'. Similarly, Hammond et al. [14] did not present such data but mentioned that all diet preparations were analyzed to confirm that they met the supplier's specifications for certified 5002 rodent diet. Monsanto [12] presented detailed data on the composition of the feed, as it is standard procedure for technical studies for regulatory feeding trials.

We conclude that the two publications by the Hammond and Séralini groups do not fully comply with this criterion while both studies mention that the composition of the different feed was measured and found to be comparable. A rejection of only one study due to the lack of reporting of data would constitute a double standard.

Open issue according to EFSA: 'The stability of the diets cannot be assessed as details of their storage conditions are not provided.'

EFSA [8] noted that Séralini et al. [3] did not inform about the storage conditions of the feed - likewise, neither Hammond et al. [14] nor Monsanto [12] informed about this. Consequently, the stability of the diets cannot be assessed in any of the studies submitted by the developers to EFSA, which went uncommented in EFSA's positive opinions on NK603 maize [16,35].

Open issue according to EFSA: 'It is impossible to evaluate whether or not there was any contamination of the diets, e.g. by mycotoxins, as it is not reported.'

Although Séralini et al. [3] did not report about mycotoxin measurements in their original publication, later, the authors [15] stated that all measured mycotoxins showed concentrations below the recommended threshold values for food and feed. The EU maximum level is 4 ppb for e.g. the sum of Aflatoxines B1, B2, G1, and G2 in processed maize for human consumption and $20 \mathrm{ppb}$ or lower for Aflatoxine B1 in feed materials. Similarly, Hammond et al. [14] informed that the levels of aflatoxins were below detection limits but did not provide the actual individual measurements or raw data. Monsanto [12] did not refer to aflatoxins alone but to the measurement of a total of 19 different mycotoxins. The author noted that there was no contamination with mycotoxins that might interfere with the results. Of the 19 analyzed mycotoxins, 4 (Deoxynivalenol, Fumonisin B1, B2, B3) could be detected in individual concentrations up $1.6 \mathrm{ppm}$ in the various maize samples. The concentration did not exceed $3 \mathrm{ppm}$ in the formulated diet, which Monsanto [12] regards as the No Observed Effect Level for Fumonisin B1 in rats according to Voss et al. [36]. The EU maximum level for Fumonisin B1 and B2 lies at $60 \mathrm{ppm}$ for maize as feed material while the EU maximum level for maize products for human consumption is 1 ppm or lower.

\section{Open issue according to EFSA: 'The amount of residues of glyphosate and its metabolites in treated maize NK603 is not reported.'}

Séralini et al. [3] did not present the results of their glyphosate measurements but stated that pesticide content did not exceed standard limits. The current EU maximum residue level for glyphosate in maize is $1 \mathrm{ppm}$. Hammond et al. [14] noted that the levels of glyphosate were below detection limits but also did not give any data. In contrast, Monsanto [12] informed that glyphosate was indeed measurable: 'Glyphosate residue in the test grain $(0.09 \mathrm{ppm})$ was slightly above the analytical detection limit of 0.05 ppm.' It is very likely that the NK603 maize used as feed was treated with a glyphosate-containing herbicide during its growth. In order to address the presence of two factors relevant for risk assessment - the transgene and the complementarily used herbicide - information on the use of glyphosate and its residues should be presented in research relevant for risk assessment [37]. 
Open issue according to EFSA: 'The exposure to $G M O, G M O+R$, and $R$ cannot be evaluated since the food and water intakes of the GM- and $R$-treated groups, respectively, are not clearly reported.'

Séralini et al. [3] did not present the values of feed and water intakes (both containing the test substances) - but also Hammond et al. [14] did not present feed intake data (only feed contained the test substance). Monsanto [12] presented feed intake data, as this is standard procedure for technical studies for regulatory feeding trials. However, this data still cannot be used to quantify the exposure to the CP4 EPSPS protein because Monsanto [12] did not quantify the concentration of CP4 EPSPS protein in the maize kernels used to mix into the feed. Monsanto [12] only conducted qualitative ELISA tests to confirm the presence or absence of the CP4 EPSPS protein. The impossibility to calculate exposure data based on the information given by Monsanto [12] was not flagged by EFSA in its two positive opinions on the NK603 dossier.

While the presentation of data on concentrations of contaminants is certainly useful, a rejection of the validity of the Séralini study based on the fact that only statements on such concentrations are given is debatable. And again, we conclude that applying a criterion to only one study would constitute a double standard in all four open issues discussed above.

\section{EFSA criterion: endpoint reporting}

Open issue according to EFSA: 'All collected endpoints should be reported openly and transparently.'

The reporting on endpoint data is an essential feature of regulatory studies. Monsanto [12] delivers all data as is required for such regulatory studies amounting to 1,180 pages supplementary information, most of them containing raw data. Obviously, such amount of data is unpublishable in any scientific journal. Therefore, Hammond et al. [14] provide means and standard deviations only and did not present the urine chemistry values. Séralini et al. [3] did not present comparable means and standard deviations summarizing the raw data, but more complex statistical evaluations of some selected data sets. Séralini et al. [15] announced that more data would be published in following publications.

The tenth open issue listed by EFSA actually shows a profound difference between the Séralini study and the two others. We conclude that the approach of the Séralini study deviates from general practice with regard to the reporting of results of such feeding studies; more information should be made available.

\section{Conclusions of the comparison of three feeding trials testing NK603 maize}

Our analyses as summarized in Table 1 revealed that the major difference between the studies of the French group and Monsanto lies in the presentation of the endpoint data, one of the contentious issues also in the public scientific debate. In January 2013, the French group has deposited their raw data at a notary and will give access to them if Monsanto's raw data on NK603 and glyphosate safety are accessible publically as well [38]. Shortly after this announcement, EFSA has enabled open access to the NK603 files at its web page [11]. Unfortunately, data on safety tests with glyphosate remain confidential, as it is the general rule for pesticide data.

For all other methodological criteria established by EFSA, we only found differences between the two peer-reviewed publications that did not exceed the normal range of differences exhibited by all published experiments that study a similar issue with similar protocols, including those considered for regulatory purposes. As expected and required, Monsanto's technical study presents more details than the two publications. These standards cannot be met by scientific publications as journals do not allow for publications exceeding several hundreds of pages of technical details. However, even in this extensive technical study, a whole range of criteria requested and applied by EFSA to the Séralini study and deplored as insufficient, were likewise absent or incomplete.

In conclusion, we note that applying the evaluation criteria to Séralini et al. [3] only but not to Monsanto [12] and Hammond et al. [14] constitutes a double standard.

Comparative analysis of other long-term rat feeding studies In a literature search effort, we found 21 peer-reviewed long-term studies with SD rats published in the last 20 years (Belpoggi et al. [39]; Bornhard et al. [40]; Butenhoff et al. [41]; Gámez et al. [42]; Gutiérrez et al. [43]; Hack et al. [44]; Holmberg and Eckstöm [45]; Johannsen and Levinskas [46]; Klimisch et al. [47]; Lee et al. [48]; Liang et al. [49]; Minardi et al. [50]; Morcos and Camilo [51]; Perricone et al. [52]; Perri et al. [53]; Quast [54]; Soffritti et al. [55]; Soffritti et al. [56]; Soffritti et al. [57]; Soffritti et al. [58]; Voss et al. [59]). The aim of our analysis of these studies was to find out whether the set of EFSA criteria with its 10 requirements is met by generally applied standards in scientific toxicology and carcinogenesis research. Our compilation revealed that the EFSA criteria and requirements applied to the Séralini study are hardly fulfilled by any of these publications (for details, see Additional file 1: Table S1). The only requirement that is consistently fulfilled by the 21 peer-reviewed publications is the endpoint reporting in the form of means and standard deviations. 
Table 1 Compliance with the EFSA requirements by the three studies

\begin{tabular}{|c|c|c|c|}
\hline \multirow[t]{2}{*}{ Requirement } & \multicolumn{3}{|c|}{ Level of compliance } \\
\hline & Séralini et al. [3] & Hammond et al. [14] & Monsanto [12] \\
\hline Study objectives need to be clearly stated a priori in the study protocol & Medium & Medium & Medium \\
\hline Suitable controls for all treatment groups need to be presented & No data shown (high) ${ }^{a}$ & High & High \\
\hline Biological relevance of the rat strain used should be justified & Low & Low & Low \\
\hline Measures taken to reduce the risk of bias (e.g. blinding) need to be taken & No data shown (high) ${ }^{a}$ & No data shown & No data shown \\
\hline Critical information about the diet composition need to be reported & No data shown & No data shown & High \\
\hline Details of the storage conditions of the feed need to be provided & No data shown & No data shown & No data shown \\
\hline $\begin{array}{l}\text { Contamination of the diets, e.g. by mycotoxins, pesticides etc., need to } \\
\text { be reported }\end{array}$ & No data shown (medium) ${ }^{a}$ & No data shown & High \\
\hline All collected endpoints should be reported openly and transparently & No data shown & Medium & High \\
\hline $\begin{array}{l}\text { The presented data need to ensure the calculation of exposure to the } \\
\text { test substance }\end{array}$ & No data shown & No data shown & Medium \\
\hline \multirow{2}{*}{$\begin{array}{l}\text { The sample size (power) calculation must be presented, especially when } \\
\text { the study objectives are unclear }\end{array}$} & \multirow[t]{2}{*}{ Low } & Low & Low \\
\hline & & $\begin{array}{l}\text { (but reference to } \\
\text { OECD GL408) }\end{array}$ & $\begin{array}{l}\text { (but reference to } \\
\text { OECD GL408) }\end{array}$ \\
\hline
\end{tabular}

The other requirements were only met by very few studies. Only one publication mentioned the OECD guideline the researchers adhered to in their experiments [42]. The choice of the strain was never discussed in scientific publications as required by EFSA. Only Butenhoff et al. [41] deliberated on the characteristics of $\mathrm{SD}$ rats in the context of comparing their results with studies using other strains. Only Liang et al. [49] applied a blinded evaluation in their histological analysis as asked for by EFSA. Butenhoff et al. [41] had chosen a blinded evaluation for their re-assessment of existing histopathological probes. None of the 21 studies reported about storage conditions or presented power calculations.

An interesting point with regard to the scientific and public discourse is the number of rats used in these peer-reviewed experiments. While public and scientific commentators, including EFSA [9], obviously assume that all animals tested per group and sex are in the end also used for analysis of e.g. biochemical parameters, the applicable OECD guidelines and several of the screened publications apply a different approach. As mentioned previously, the OECD Guideline 452 recommends the use of 10 out of the 20 rats per group for biochemical analysis. As indicated in (Additional file 1: Table S1) 9 of the 12 publications that analyzed biochemical parameters in the toxicology study also used 10 or fewer rats in some or all tests.

\section{EFSA reactions regarding the three NK603 studies}

Monsanto [12] reported 77 comparisons in which statistically significant differences between the NK603 treatments and the different controls were measured (for details, see Additional file 2: Table S2). The authors considered $71 \%$ (55) of the statistically significant differences between treatments and controls as either 'not biologically significant' (19), 'not biologically relevant' (28), 'not biologically meaningful' (6), or 'not toxicologically significant' (2). Seventeen of the remaining 22 comparisons showed a significantly higher food consumption of the HD 33\% NK603 maize group. Monsanto [12] noted that '[i]n total, some 1,050 comparisons were made and approximately 53 of these were anticipated to be significant by chance alone at the $5 \%$ significance level.' It remains unclear if the author intended to suggest that these remaining 22 significant differences were produced by mere chance and, thus, could also be regarded as irrelevant. The author's decisions are based on conceptual and methodological arguments, no peer-reviewed science is provided to underpin the argumentation and categorization.

In its first opinion - based on Monsanto [12] - EFSA [35] did neither report about the 77 statistically significant differences nor about the explanations of Monsanto and the underlying norms and standards to declare 55 of them as irrelevant. In fact, no agreed EU norms and standards existed in 2003 that EFSA could have used to justify such a categorization. EFSA also did not comment on the remaining 22 comparisons that Monsanto did not declare explicitly as insignificant but did so implicitly by assigning them to pure chance only and, thus, did forego any further analysis as to their quality. EFSA declared that only two comparisons - average corpuscular volume and average corpuscular haemoglobin in female rats in the high-dose group - exhibiting 'slightly elevated levels' showed 'consistent differences' 
(Table 2). It remains unclear why only these two 'slightly' differing comparisons out of 77 significant differences were singled out and much more pronounced differences recorded, for example, in the group of haematology values went uncommented by EFSA.

In its second opinion, EFSA [16] based its essentially unchanged conclusions on Hammond et al. [14]. This is surprising because the authors state explicitly that there are no statistically significant differences anymore. The reason for this complete loss of the statistical significant differences still reported by Monsanto [12] is due to a new approach of dealing with the raw data. In contrast to Monsanto [12], two modifications were applied: (1) the only comparator in Hammond et al. [14] are the mean values of all six reference controls but not of the parental control and, (2) additionally, the double standard deviation is used as upper and lower threshold to determine the statistical significance. This new statistical approach - uncommented by EFSA - resulted in eliminating all statistically significant differences as still reported by Monsanto [12]. No scientifically documented justification for this approach to eliminate statistical differences was provided by Monsanto nor requested by EFSA.

In conclusion, with regard to the provisions of the EU laws on GM organism risk assessment and approval, we argue that a comparison of a GM plant only to genetically unrelated reference controls excluding the unmodified isogenic parents is not in compliance with EU law and guidelines on GMO risk assessment. Based on the above reported outcomes of our comparative analysis, we note a striking double standard in rigor applied in the evaluation of the submitted rat feeding trials conducted and submitted by the developer to EFSA in comparison to the rigor applied in the evaluation of the Séralini study.

\section{The need for appropriate regulatory sciences}

Of prime importance and long overdue is an inclusive discussion on the appropriate testable, scientific hypotheses and methodologies that are supportive of the provisions and tasks formulated by the EU legal framework on risk assessment of GM organisms. The EU law is based on the axiom that risks, which may be caused by GM organisms, cannot be a priori-deducted from experience with existing organisms. Due to their genomic and evolutionary novelty, specific laws regulate the testing in the open environment and the market approval process of GM organisms since 1990. Therefore, the EU Directive $2001 / 18$ on the deliberate release into the environment of genetically modified organisms clearly states as a 'General Principle' for environmental risk assessment that 'identified characteristics of the GMO and its use which have the potential to cause adverse effects should be compared to those presented by the nonmodified organism from which it is derived and its use under corresponding situations'. Nowhere does this Directive or the Regulation (EC) No 1829/2003 on genetically modified food and feed suggest that GM organisms also need to be compared to unrelated control organisms in the context of risk assessment (see Regulation

Table 2 Comparison of EFSA's scientific opinions on Monsanto's rat feeding study with NK603 maize

\section{Safety of the whole GM food/feed ${ }^{\text {a }}$}

Sub-chronic (90-days) toxicity studies in rats fed maize NK603.

No consistent differences in the measured clinical, biochemical, and histological parameters were noted for rats fed on non-GM or NK603 maize, except for slightly elevated levels of average corpuscular volume and average corpuscular haemoglobin in female rats administered with a high dose.

Since both parameters were calculated from other data (hematocrit/red blood cells and haemoglobin concentration/red blood cells, respectively), and no other observations of treatment-related effects were made, the applicant suggests that an artifactual difference resulted from a slightly higher hematocrit or haemoglobin concentration and slightly lower red blood cell count at these sampling points.

Furthermore, the applicant concludes that these findings are of no biological significance.

The Panel accepts this as a reasonable interpretation of the data. The Panel also found the doses chosen for the study (11\% or 33\% of diet) appropriate, as they did not distort the nutritional balance of the experimental animals. The standard rodent diet used by the test laboratory contains approximately 33\% maize grain.

\section{Toxicological assessment of the whole GM food/feed ${ }^{b}$}

A published 90 -day study in rats fed either maize NK603 as 11\% or 33\% of the diet, or a diet which to $11 \%$ or $33 \%$ was made up of non-GM maize grain having a comparable genetic background to maize NK603 (LH82 x B73), resulted in

no consistent differences in the measured clinical, biochemical, and histological parameters, except for slightly elevated levels of average corpuscular volume and average corpuscular haemoglobin in female rats administered the high dose (Hammond et al., 2004).

Since both parameters are calculated (hematocrit/red blood cells and haemoglobin concentration/red blood cells, respectively), and no other observations of treatment-related effects were made, the applicant suggested that these statistical differences were artefacts resulting from a slightly higher hematocrit or haemoglobin concentration and slightly lower red blood cell count at this sampling.

Furthermore, it was pointed out that the observed difference in average corpuscular volume and average corpuscular haemoglobin had no biological relevance.

The EFSA GMO Panel finds the interpretation of the data acceptable. The EFSA GMO Panel also found the doses chosen for the study $(11 \%$ or $33 \%$ of diet) appropriate, as they did not distort the nutritional balance of the experimental animals. The standard rodent diet used by the test laboratory contains approximately 33\% maize grain. 
1829/2003 Art. 4 1. and Art. 5 3. (e)). The evaluation of characteristics of a GM organism with regard to the 'accepted limits of natural variations' - requiring data from unrelated reference varieties - is only mentioned in Art. 53. (f) in the context of labelling but not risk assessment.

We argue that the concept of substantial equivalence or the concept of familiarity, originally developed in the context of the US regulatory system for GM organisms and based on opposite assumptions as the EU regulatory system [60], is not per se applicable in the EU context. One element of this US approach is the additional use of unrelated varieties as reference controls to establish the natural variation against which GM food products need to be evaluated. While this approach was taken up in a report by the World Health Organisation [61] and in a technical risk assessment guidelines of the Codex Alimentarius Commission [62], it failed to become recognized in the more general Codex Alimentarius Commission Principles 44-2003 [63] or in the international legally binding Cartagena Protocol on Biosafety [64]. In practice, adding genetically unrelated controls will increase the unspecific background variability in the data and make the statistical detection of specific differences due to the insertion of the transgenic trait more difficult or impossible.

We conclude that the actual effect of introducing the use of unrelated reference controls lies more in providing an instrument to declare technology-induced significant differences as irrelevant against the background of unspecified 'noise' (stemming from broad, unrelated natural variation) than in setting up a sound methodology for risk assessment under the applicable legal framework. While over the past years, no norms, standards, and scientific criteria for the concept of familiarity and its core instrument of reference controls were developed, it is still at the discretion of applicants and authorities if and how to use the respective test results.

The non-scientific character of this approach can be illustrated through its use by Monsanto [12]. Monsanto selected six maize varieties as reference controls 'selected to represent a range of growing environments and a diversity of germplasm'. Monsanto does not prove that the selected varieties and the growing areas are indeed representative for the USA maize production and subsequent exposure of animals and humans to maize products. Such demonstrated representativeness would be essential to justify the use of reference control as means to declare biological irrelevance in regulatory science, as for example suggested by the Codex Alimentarius Commission guidelines [62]. EFSA accepted the approach by Monsanto [12] without requiring proof for representativeness or any other robust scientific justification of the use of these reference controls. In order to obtain some information about the six varieties, we performed an internet search. As shown in Table 3, no relevant
Table 3 Information on relevance of reference varieties used by Monsanto [12] for the US corn production

\begin{tabular}{ll}
\hline Reference variety & Summary of internet search \\
\hline Campbells 6995 & No relevant information available \\
Cropplan Genetics 461 & No relevant information available \\
Crows 363 & No relevant information available \\
DK537 & Indication of use ten and more years ago \\
& Parental line of Yield Gard rootworm corn \\
& Dekalb DKC53-29 \\
Indication of use ten and more years ago & Described as 'widely adapted' by Dekalb \\
DK539 & in 1999 \\
& Highly successful corn variety until 1995, \\
& dominating the US corn belt \\
Pioneer 3394 & $\begin{array}{l}\text { After causing high economic losses in 95/96 } \\
\text { due to very low resistance to Gray leaf spot } \\
\text { fungi, the planting of this variety ceased }\end{array}$
\end{tabular}

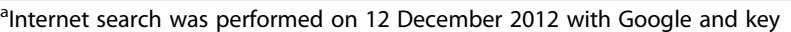
words < 'name' AND corn > as well as < 'name' AND corn AND acreage >.

information could be found for three of the used varieties. Two of them obviously had some level of importance ten or more years ago. Only for Pioneer 3394 maize, we were able to retrieve information that showed high relevance of this variety for the US maize agriculture in the 1990s. Based on this search, we conclude that it is doubtful whether the chosen six varieties were a representative sample for the US maize when the Monsanto rat study was performed. In any case, it can safely be concluded that the six varieties were of no relevance for EU maize agriculture when EFSA accepted the study in 2003 and 2009. Biological irrelevance of many statistically significant differences between NK603 maize and its unmodified parents was declared without any scientific justification or experimental proof.

\section{Conclusions}

The recent controversy focused on the validity of the study published by Séralini et al. [3] as adequate evidence for questioning the safety of NK603 maize and its glyphosate-based co-technology. In our comparative analysis based on four out of five criteria of EFSA, we found the studies of Monsanto [12] and Hammond et al. [14] submitted as evidence for proving the safety of NK603 maize to suffer from comparable deficiencies. We did find critical double standards in acceptance and rigor of the evaluation of feeding studies submitted as proof of safety for regulatory approval to EFSA. We further conclude that during 23 years of EU regulation for GM organisms and various GM crop risk assessments and approvals, regulatory sciences and methodology are used that were developed under the contrasting US regulatory concept. It is this mismatch between these different legal frameworks and the choice of risk 
assessment methodology that we see to be the basis of the often-stated public mistrust in the EU authorities. Only recently, the EU authorities started to build up an implementing system that takes its directives from the current EU legislation and is supportive of its own approach. Concerns about the intransparent and arbitrary use of reference controls as instrument to state biological irrelevance of significant differences between GM organisms and its parents must be addressed in these ongoing activities. Activities of prime concern in this regard is the GMO Risk Assessment and Communication of Evidence (GRACE) Project [65] developing protocols for 90-day rat feeding trials with GM plants and certainly influencing the suggested development of respective 2-year testing protocols with rats. But until now, the GRACE Project adheres to the intransparent use of reference varieties. Until the scientific questions with regard to appropriate risk research and assessment are resolved and the application of double standards has ceased, we do not expect that neither the public nor the scientific debate will subside.

\section{Additional files}

Additional file 1: Analysis of 21 long-term toxicity and/or carcinogenicity studies with SD rats according to selected EFSA criteria.

Additional file 2: Summary of statistically significant differences between test and controls groups in Monsanto [12].

\section{Abbreviations}

CP4 EPSPS: 5-enolpyruvylshikimate-3-phosphate synthase of agrobacterium strain CP4; HD: High dose; LD: Low dose; SD: Sprague-Dawley (rats).

\section{Competing interests}

The authors declare that they have no competing interests. The work of HM was financially supported by ENSSER. ENSSER did not have any role in study design; in the collection, analysis, and interpretation of data; in the writing of the report; and in the decision to submit the paper for publication.

\section{Authors' contributions}

HM carried out the analysis of the documents submitted to EFSA by Monsanto with its application for approval of NK602 maize for food and feed purposes. HM and AH wrote the review. Both authors read and approved the final manuscript.

\section{Acknowledgements}

The authors thank several ENSSER members for initial discussions and valuable comments.

\section{Author details}

${ }^{1}$ European Network of Scientists for Social and Environmental Responsibility (ENSSER), In den Steinäckern 13, 38116 Braunschweig, Germany. ${ }^{2}$ Swiss Federal Institute of Technology, Institute of Integrative Biology IBZ, Universitätstrasse, 16, 8092 Zurich, Switzerland.

Received: 15 August 2013 Accepted: 18 November 2013 Published: 1 December 2013

\section{References}

1. Séralini GE, Cellier D, Spiroux de Vendômois J: New analysis of a rat feeding study with a genetically modified maize reveals signs of hepatorenal toxicity. Arch Environ Contam Toxicol 2007, 52:596-602.

2. European Commission: Commission Implementing Regulation (EU) No 503/2013 of 3 April 2013 on applications for authorisation of genetically modified food and feed in accordance with Regulation (EC) No 1829/ 2003 of the European Parliament and of the Council and amending Commission Regulations (EC) No 641/2004 and (EC) No 1981/2006. Offic J EU 2013, L157(56):1-48.

3. Séralini GE, Clair E, Mesnage R, Gress S, Defarge N, Malatesta M, Hennequin D, Spiroux de Vendômois J: Long term toxicity of a Roundup herbicide and a Roundup-tolerant genetically modified maize. Food Chem Toxicol 2012, 50:4221-4231.

4. Arjó G, Portero M, Piñol C, Viñas J, Matias-Guiu X, Capell T, Bartholomaeus A, Parrott W, Christou P: Plurality of opinion scientific discourse and pseudoscience: an in depth analysis of the Séralini et al. study claiming that Roundup Ready corn or the herbicide Roundup cause cancer in rats. Transgen Res 2013, 22:255-267.

5. Nicole W: A closer look at GE corn findings. Environ Health Perspec 2012, 120(11):A421.

6. Robinson C, Holland N, Leloup D, Muilerman H: Conflicts of interest at the European Food Safety Authority erode public confidence. J Epidemiol Community Health 2013, 67(9):717-720.

7. Anonymus: Commission and EFSA agree need for two-year GMO feeding studies. EU Food Policy 2012, 17:12-2012.

8. European Food Safety Authority: Review of the Séralini et al. (2012) publication on a 2-year rodent feeding study with glyphosate formulations and GM maize NK603 as published online on 19 September 2012 in Food and Chemical Toxicology. EFSA J 2012, 10(10):2910.

9. EFSAS Committee: EFSA guidance on conducting repeated-dose 90-day oral toxicity study in rodents on whole food/feed. EFSA J 2011, 9(12):2438.

10. European Food Safety Authority: EFSA promotes public access to data in transparency initiative. Parma: Press Release; 2013. 14.01.

11. Monsanto: Dossier for NK603 maize approval at EFSA. http://www.efsa. europa.eu/en/press/news/130114.htm

12. Monsanto: 13 week feeding study in rats with grain from Roundup Ready corn (NK603) preceded by a 1-week baseline food consumption determination with PMI Certified Rodent Diet \#5002. St. Louis; 2001.

13. European Food Safety Authority: Final review of the Séralini et al. (2012) publication on a 2-year rodent feeding study with glyphosate formulations and GM maize NK603 as published online on 19 September 2012 in Food and Chemical Toxicology. EFSA J 2012, 10(11):2986.

14. Hammond B, Dudek R, Lemen J, Nemeth M: Results of a 13 week safety assurance study with rats fed grain from glyphosate tolerant corn. Food Chem Toxicol 2004, 42:1003-1014.

15. Séralini GE, Mesnage R, Defarge N, Gress S, Hennequin D, Clair E, Malatesta M, Spiroux de Vendômois J: Answers to critics: why there is a long term toxicity due to a Roundup-tolerant genetically modified maize and to a Roundup herbicide. Food Chem Toxicol 2013, 53:476-483.

16. European Food Safety Authority Panel on Genetically Modified Organisms: Applications (references EFSA-GMO-NL-2005-22 EFSA-GMO-RX-NK603) for the placing on the market of the genetically modified glyphosate tolerant maize NK603 for cultivation food and feed uses import and processing and for renewal of the authorisation of maize NK603 as existing products both under Regulation (EC) No 1829/2003 from Monsanto. Scientific Opinion of the Panel on Genetically Modified Organisms. EFSA J 2009, 1137:1-50.

17. European Commission: New study entitled "Long term toxicity of a Roundup herbicide and a Roundup-tolerant genetically modified maize", Letter of EC DG SANCO to EFSA of 26.09.2012. http://registerofquestions.efsa.europa.eu/ rogFrontend/questionLoader?question=EFSA-Q-2012-00841.

18. Organization for Economic Cooperation and Development: OECD guideline for testing chemicals 408 - Repeated dose 90-day oral toxicity study in rodents. Paris; 1998.

19. Organization for Economic Cooperation and Development: OECD guideline for testing chemicals 453 - Combined chronic toxicity Icarcinogenicity studies. Paris; 2009

20. Organization for Economic Cooperation and Development: OECD guideline for testing chemicals 452 - Chronic toxicity studies. Paris; 2009.

21. Organization for Economic Cooperation and Development: OECD guideline for testing chemicals 451 - Carcinogenicity studies. Paris; 2009.

22. Organization for Economic Cooperation and Development: OECD guideline for testing chemicals 452 - Chronic toxicity studies. Paris; 1981.

23. King-Herbert AP, Sills RC, Bucher JR: Commentary: update on animal models for NTP studies. Toxicol Pathol 2010, 38:180-181.

24. Soffritti M, Belpoggi F, Minardi F, Maltoni C: History and major projects lifespan carcinogenicity bioassay design chemicals studied and results. Ann N Y Acad Sci 2002, 982:26-45. 
25. Science Media Centre: Study on cancer and GM maize - experts respond. Science Alert of 20.09.2012. http://www.sciencemediacentre.co.nz/2012/09/ 20/study-on-cancer-and-gm-maize-experts-respond/.

26. Organization for Economic Cooperation and Development, Organization for Economic Cooperation and Development: Guidance document 116 on the conduct and design of chronic toxicity and carcinogenicity studies supporting Test Guidelines 451452 and 453. Paris; 2012.

27. Unites States Food and Drug Authority: Redbook 2000 - Toxicological Principles for the Safety Assessment of Food Ingredients. Chapter N.C.5.a. Chronic Toxicity Studies with Rodents p.142-153 \& Chapter IV.C.6, Carcinogenicity Studies with Rodents. College Park; 2007:164-175.

28. Dinse GE, Peddada SD, Harris SF, Elmore SA: Comparison of NTP historical control tumor incidence rates in female Harlan Sprague Dawley and Fischer 344/N rats. Toxicol Pathol 2010, 38:765-775.

29. Giknis MLA, Clifford CB: Compilation of Spontaneous Neoplastic Lesions and Survival in Crl CD (SD) Rats from Control Groups. Charles River Laboratories: Wilmington; 2004.

30. Keenan KP, Ballam GC, Dixit R, Soper KA, Laroque P, Mattson BA, Adams SP, Coleman JB: The effects of diet overfeeding and moderate dietary restriction on Sprague-Dawley rat survival disease and toxicology. J Nutr 1997, 127:852S-856S.

31. Alemán CL, Más RM, Rodeiro I, Noa M, Hernández C, Menéndez R, Gámez R: Reference database of the main physiological parameters in SpragueDawley rats from 6 to 32 months. Lab Anim 1998, 32:457-466.

32. Draft for public consultation - Scientific Opinion EFSA quidance on repeateddose 90-day oral toxicity study on whole food/feed in rodents. http://www. efsa.europa.eu/en/consultationsclosed/call/110707.pdf.

33. European Food Safety Authority: Outcome of the public consultation on the draft scientific opinion on EFSA guidance on repeated dose 90-day oral toxicity study on whole food/feed in rodents. Supporting Publications 2011:205.

34. Crissman JW, Goodman DG, Hildebrandt PK, Maronpot RR, Prater DA, Riley $\mathrm{JH}$, Seaman WJ, Thake DC: Best practices guideline: toxicologic histopathology. Toxicol Pathol 2004, 32:126-131.

35. European Food Safety Authority Panel on Genetically Modified Organisms: Opinion of the scientific panel on genetically modified organisms on a request from the commission related to the safety of foods and food ingredients derived from herbicide-tolerant genetically modified maize NK603 for which a request for placing on the market was submitted under Article 4 of the Novel Food Regulation (EC) No 258/97 by Monsanto (QUESTION NO EFSA-Q-2003-002). EFSA J 2003, 9:1-14.

36. Voss KA, Chamberlain WJ, Bacon CW, Riley RT, Norred WP: Subchronic toxicity of fumonisin B 1 to male and female rats. Fundam Appl Toxicol 1995, 24:102-110.

37. Viljoen C: Letter to the Editor. Food Chem Toxicol 2013, 59:809-810.

38. CRIIGEN: Toxicity confirmed for a GMO and the pesticide Roundup. Caen: Press Release; 2013. 11.01

39. Belpoggi F, Soffritti M, Minardi F, Bua L, Cattin E, Maltoni C: Results of longterm carcinogenicity bioassays on tert-amyl-methyl-ether (TAME) and di-isopropyl-ether (DIPE) in rats. Ann N Y Acad Sci 2002, 982:70-86.

40. Bornhard E, Bischoff $H$, Mager $H$, Krötlinger F, Schilde B: D-Glucose combined chronic toxicity and carcinogenicity studies in Sprague-Dawley rats and Syrian golden hamsters. Drug Chem Toxicol 1998, 21(3):329-353.

41. Butenhoff JL, Kennedy GL Jr, Chang SC, Olsen GW: Chronic dietary toxicity and carcinogenicity study with ammonium perfluorooctanoate in Sprague-Dawley rats. Toxicology 2012, 298:1-13.

42. Gámez R, Noa M, Mas R, Mendoza N, Pardo B, Menéndez R, Pérez $Y$, González RM, Gutiérrez A, Marrero G, Goicochea E, García H, Curveco D: Long-term carcinogenicity of D-003 a mixture of high molecular weight acids from sugarcane wax in Sprague Dawley rats: a 24 months study. Food Chem Toxicol 2007, 45:2352-2358.

43. Gutiérrez A, Gámez R, Noa M, Mas R, Arencibia D, Pardo B, Valle M, Oyarzábal A, Curveco D, García H, Goicochea E, Mendoza M, Jiménez S: One year oral toxicity of D-004 a lipid extract from Roystonea regia fruits in Sprague Dawley rats. Food Chem Toxicol 2011, 49:2855-2861.

44. Hack R, Ebert E, Leist KH: Chronic toxicity and carcinogenicity studies with the insecticide endosulfan in rats and mice. Food Chem Toxicol 1995, 33(11):941-950

45. Holmberg B, Eckstöm T: The effects of long-term oral administration of ethanol on Sprague-Dawley rats - a condensed report. Toxicology 1994, 96:133-145.

46. Johannsen FR, Levinskas GJ: Comparative chronic toxicity and carcinogenicity of acrylonitrile by drinking water and oral intubation to Spartan Sprague-Dawley rats. Toxicol Lett 2002, 132:197-219.
47. Klimisch HJ, Deckardt K, Gembardt C, Hildebrand B, Küttler K, Roe FJC: Long-term inhalation toxicity of $\mathrm{N}$-vinylpyrrolidone-2 vapours. Studies in rats. Food Chem Toxicol 1997, 35:1041-1060

48. Lee HJ, Gimm YM, Choi HD, Kim N, Kim SH, Lee YS: Chronic exposure of Sprague-Dawley rats to $20 \mathrm{kHz}$ triangular magnetic fields. Int J Radiat Biol 2010, 86(5):384-389.

49. Liang J, Pei X, Zhang Z, Wang N, Wang J, Li Y: The protective effects of long-term oral administration of marine collagen hydrolysate from chum salmon on collagen matrix homeostasis in the chronological aged skin of Sprague-Dawley male Rats. J Food Sci 2010, 75(8):H230-H238.

50. Minardi F, Belpoggi F, Soffritti M, Ciliberti A, Lauriola M, Cattin E, Maltoni C: Results of long-term carcinogenicity bioassay on vinyl acetate monomer in Sprague-Dawley rats. Ann N Y Acad Sci 2002, 982:106-122

51. Morcos NC, Camilo K: Acute and chronic toxicity study of fish oil and garlic combination. Int J Vitam Nutr Res 2001, 71(5):306-312.

52. Perricone NV, Bagchi D, Echard B, Preuss HG: Long-term metabolic effects of different doses of niacin-bound chromium on Sprague-Dawley rats. Mol Cell Biochem 2010, 338:91-103.

53. Perri GC, Nunziata A, Argentino-Storino A, Salerno RO, Mercatelli P: Long term toxicity and carcinogenicity of a new protein source in rats. Toxicol Eur Res 1981, 3(6):305-310.

54. Quast JF: Two-year toxicity and oncogenicity study with acrylonitrile incorporated in the drinking water of rats. Toxicol Lett 2002, 132:153-196.

55. Soffritti M, Belpoggi F, Cevolani D, Guarino M, Padovani M, Maltoni C: Results of long-term experimental studies on the carcinogenicity of methyl alcohol and ethyl alcohol in rats. Ann N Y Acad Sci 2002, 982:46-69.

56. Soffritti M, Belpoggi F, Degli Esposti D, Lambertini L, Tibaldi E, Rigano A: First experimental demonstration of the multipotential carcinogenic effects of aspartame administered in the feed to Sprague-Dawley rats. Environ Health Perspect 2006, 114(3):379-385.

57. Soffritti M, Belpoggi F, Degli Esposti D, Lambertini L: Results of a long-term carcinogenicity bioassay on Sprague-Dawley rats exposed to sodium arsenite administered in drinking water. Ann N Y Acad Sci 2006, 1076:578-591

58. Soffritti M, Belpoggi F, Tibaldi E, Degli Esposti D, Lauriola M: Life-span exposure to low doses of aspartame beginning during prenatal life increases cancer effects in rats. Environ Health Perspect 2007, 115(9):1293-1297.

59. Voss C, Zerban H, Bannasch P, Berger MR: Lifelong exposure to di-(2-ethylhexyl)phthalate induces tumors in liver and testes of Sprague-Dawley rats. Toxicology 2005, 206:359-371.

60. Meyer $\mathrm{H}$ : Systemic risks of genetically modified crops the need for new approaches to risk assessment. Environ Sci Eur 2011, 23:7.

61. World Health Organization: Application of the principles of substantial equivalence to the safety evaluation of foods or food components from plants derived by modern biotechnology. Geneva: Report of a WHO workshop; 1995.

62. Codex Alimentarius Commission: Guideline for the conduct of food safety assessment of foods derived from recombinant-DNA plants. Rome: Food and Agriculture Organization; 2003.

63. Codex Alimentarius Commission: Principles for the risk analysis of foods derived from modern biotechnology. Rome: Food and Agriculture Organization; 2003

64. Convention on Biological Diversity: Cartagena Protocol on Biosafety to the Convention on Biological Diversity. Montreal; 2000

65. GMO Risk Assessment and Communication of Evidence (GRACE) Project. http://www.grace-fp7.eu/

doi:10.1186/2190-4715-25-33

Cite this article as: Meyer and Hilbeck: Rat feeding studies with genetically modified maize - a comparative evaluation of applied methods and risk assessment standards. Environmental Sciences Europe 2013 25:33. 